\title{
Present status and future of ultrasonic quantitative diagnostic techniques
}

\author{
Hiroyuki Hachiya ${ }^{1}$
}

(c) The Japan Society of Ultrasonics in Medicine 2018

Great results are being achieved with diagnosis using ultrasound-based medical imaging, and the image quality of ultrasonic diagnostic equipment continues to improve year after year. New quantitative techniques using shear waves are also becoming available in addition to conventional interpretation of images. There are attempts being made in various regions aimed at acquiring quantitative diagnostic information by utilizing changes in acoustic properties $[1,2]$, and it is hoped that these efforts will lead to further advances.

On the other hand, the quantitative relationship between various lesions and changes in ultrasonic signals is not necessarily clear. Biological tissue and biological functions are complex, and it is not possible to directly observe the majority of phenomena in vivo. As such, there is rarely a clear one-to-one correspondence between cause and result. Therefore, various statistical procedures are often used in the assessment of diagnostic or therapeutic approaches. This type of statistical assessment is logical from a medical standpoint, but from an engineering standpoint, it can lead to obfuscation of where the issue lies.

To understand changes in ultrasonic signals caused by lesions, when investigating such changes it is important to clearly distinguish between (a) changes in biological tissue caused by a lesion, (b) changes in the acoustic properties of biological tissue, and (c) the relationship between tissue acoustic changes and the ultrasonic signal. If a systematic and quantitative investigation progresses from determining the degree of change in the acoustic properties relative to the change in biological tissue caused by a lesion $[(a) \rightarrow(b)]$ to then determining the degree of change in the propagating ultrasonic wave relative to the change in acoustic properties $[(b) \rightarrow(c)]$, it will be possible to acquire quantitative information on the change in biological tissue based on the change in the ultrasonic signal acquired from the patient $[(\mathrm{c}) \rightarrow(\mathrm{a})]$.

Ultrasonic microscopes that measure the minute structure of the acoustic properties of biological tissue have advanced to a level where it is now possible to identify individual cells, which has yielded new findings about in vivo elastic characteristics. Advances in equipment have made it possible to perform complex processing of ultrasonic signals, which has allowed development of more sophisticated quantitative diagnosis. The Japan Society of Ultrasonics in Medicine, where members can interact closely to discuss ideas based on a mutual understanding between those in the medical and engineering disciplines, plays a central role in such technological advances. I hope that your active submissions will lead to further dissemination of new technologies via this journal.

\section{References}

1. Otani T, Fukunaga M, Yho K, et al. Attempt at standardization of bone quantitative ultrasound in Japan. J Med Ultrason. 2018;45:313. https://doi.org/10.1007/s10396-017-0814-5.

2. Isono H, Hirata S, Yamaguchi T, et al. Analysis of fluctuation for pixel-pair distance in co-occurrence matrix applied to ultrasonic images for diagnosis of liver fibrosis. J Med Ultrason. 2017;44:23-35. https://doi.org/10.1007/s10396-016-0741-x.
Hiroyuki Hachiya

hachiya@ctrl.titech.ac.jp

1 Department of Systems and Control Engineering, School of Engineering, Tokyo Institute of Technology,

Tokyo 152-8550, Japan 